\title{
Exact Solution for an Anti-Plane Interface Crack between Two Dissimilar Magneto-Electro-Elastic Half-Spaces
}

\author{
Bogdan Rogowski \\ Department of Mechanics of Materials, Technical University of Lodz, Al. Politechniki 6, 93-590 Lodz, Poland \\ Correspondence should be addressed to Bogdan Rogowski, bogdan.rogowski@p.lodz.pl
}

Received 27 October 2011; Accepted 10 February 2012

Academic Editor: Ma Jan

Copyright () 2012 Bogdan Rogowski. This is an open access article distributed under the Creative Commons Attribution License, which permits unrestricted use, distribution, and reproduction in any medium, provided the original work is properly cited.

This paper investigated the fracture behaviour of a piezo-electro-magneto-elastic medium subjected to electro-magnetomechanical loads. The bimaterial medium contains a crack which lies at interface and is parallel to their poling direction. Fourier transform technique is used to reduce the problem to three pairs of dual integral equations. These equations are solved exactly. The semipermeable crack-face magneto-electric boundary conditions are utilized. Field intensity factors of stress, electric displacement, magnetic induction, cracks displacement, electric and magnetic potentials, and the energy release rate are determined. The electric displacement and magnetic induction of crack interior are discussed. Obtained results indicate that the stress field and electric and magnetic fields near the crack tips exhibit square-root singularity.

\section{Introduction}

Mechanics of magneto-electro-elastic solid has gained considerable interest in the recent decades with increasable wide application of piezoelectric/piezomagnetic composite materials in engineering, particularly in aerospace and automotive industries. Recently, much attention has been paid to dislocation crack and inclusion problems in magneto-electroelastic solids, which simultaneously possess piezoelectric, piezomagnetic, and magnetoelectric effects. Therefore, it is of vital importance to investigate the magneto-electro-elastic fields as a result of existence of defects, such as cracks, in these solids.

The mode III interface crack solution for two dissimilar half-planes has been analyzed by Li and Kardomateas [1] while for two dissimilar layers by Wang and Mai [2] and by Li and Wang [3]. Li and Kardomateas [1] solved corresponding plane problem by means of Stroh's formalism and complex variable methods. Li and Wang [3] investigated the problems involving an antiplane shear crack perpendicular to and terminating at the interface of a bimaterial piezo-electroelastic ceramics. Wang and Mai [2] investigated the mode III-crack problem for a crack at the interface between two dissimilar magneto-electro-elastic layers. Extension of those investigation problems interested in fracture theory, on dielectric and magnetostrictive crack behaviour, is very important, and results and conclusions could have applications in the failure of PEMO-elastic devices and in smart intelligent structures [4].

However, to the authors' best knowledge, no researches dealing with the interface-dielectric crack in PEMO-elastic two-phase composite have been reported in literature. When subjected to mechanical, electrical, and magnetic loads in service, these magneto-electro-elastic composites can fail prematurely due to some defects, namely, cracks, holes and others, arising during their manufacturing process. Therefore, it is of great importance to study the magnetoelectro-elastic interaction and fracture behaviours of PEMOelastic materials.

In mechanic where two-phase composites have twelve material constants only exact solutions are useful. This is motivation for this study. For electrical, magnetic, and mechanical loads (two cases of electrical and magnetic excitations) and semipermeable electrical and magnetic boundary conditions at the interface crack, exact analytical solutions are obtained here for full field interesting in fracture mechanics. 


\section{Basic Equations}

For a linearly magneto-electro-elastic medium under antiplane shear coupled with in-plane electric and magnetic fields, there are only the nontrivial antiplane displacement $w$

$$
u_{x}=0, \quad u_{y}=0, \quad u_{z}=w(x, y)
$$

strain components $\gamma_{x z}$ and $\gamma_{y z}$

$$
\gamma_{x z}=\frac{\partial w}{\partial x}, \quad \gamma_{y z}=\frac{\partial w}{\partial y}
$$

stress components $\tau_{x z}$ and $\tau_{y z}$, in-plane electrical and magnetic potentials $\phi$ and $\psi$, which define electrical and magnetic field components $E_{x}, E_{y}, H_{x}$ and $H_{y}$

$$
\begin{array}{ll}
E_{x}=-\frac{\partial \phi}{\partial x}, & E_{y}=-\frac{\partial \phi}{\partial y}, \\
H_{x}=-\frac{\partial \psi}{\partial x}, & H_{y}=-\frac{\partial \psi}{\partial y}
\end{array}
$$

and electrical displacement components $D_{x}, D_{y}$, and magnetic induction components $B_{x}, B_{y}$ with all field quantities being the functions of coordinates $x$ and $y$.

The generalized strain-displacement relations (2) and (3) have the form

$$
\gamma_{\alpha z}=w_{, \alpha}, \quad E_{\alpha}=-\phi_{, \alpha}, \quad H_{\alpha}=-\psi_{, \alpha},
$$

where $\alpha=x, y$ and $w_{, \alpha}=\partial w / \partial \alpha$.

For linearly magneto-electro-elastic medium, the coupled constitutive relations can be written in the matrix form

$$
\left[\tau_{\alpha z}, D_{\alpha}, B_{\alpha}\right]^{T}=C\left[\gamma_{\alpha z},-E_{\alpha},-H_{\alpha}\right]^{T},
$$

where the superscript $T$ denotes the transpose of a matrix, and

$$
C=\left[\begin{array}{ccc}
c_{44} & e_{15} & q_{15} \\
e_{15} & -\varepsilon_{11} & -d_{11} \\
q_{15} & -d_{11} & -\mu_{11}
\end{array}\right]
$$

is the material property matrix, where $c_{44}$ is the shear modulus along the $z$-direction, which is direction of poling and is perpendicular to the isotropic plane $(x, y), \varepsilon_{11}$ and $\mu_{11}$ are dielectric permittivity and magnetic permeability coefficients, respectively, $e_{15}, q_{15}$, and $d_{11}$ are piezoelectric, piezomagnetic, and magneto-electric coefficients, respectively.

The mechanical equilibrium equation (called as Euler equation), the charge and current conservation equations (called as Maxwell equations), in the absence of the body force electric and magnetic charge densities, can be written as

$$
\tau_{z \alpha, \alpha}=0, \quad D_{\alpha, \alpha}=0, \quad B_{\alpha, \alpha}=0, \quad \alpha=x, y .
$$

Subsequently, the Euler and Maxwell equations take the form

$$
C\left[\nabla^{2} w, \nabla^{2} \phi, \nabla^{2} \psi\right]^{T}=[0,0,0]^{T},
$$

where $\nabla^{2}=\partial^{2} / \partial x^{2}+\partial^{2} / \partial y^{2}$ is the two-dimensional Laplace operator.

Since $|C| \neq 0$, one can decouple $(8)$

$$
\nabla^{2} w=0, \quad \nabla^{2} \phi=0, \quad \nabla^{2} \psi=0 .
$$

If we introduce, for convenience of mathematics in some boundary value problems, two unknown functions

$$
\left[\chi-e_{15} w, \eta-q_{15} w\right]^{T}=C_{0}[\phi, \psi]^{T},
$$

where the matrix $C_{0}$, a principal submatrix of $C$, is

$$
C_{0}=\left[\begin{array}{ll}
-\varepsilon_{11} & -d_{11} \\
-d_{11} & -\mu_{11}
\end{array}\right],
$$

then

$$
[\phi, \psi]^{T}=C_{0}^{-1}\left[\chi-e_{15} w, \eta-q_{15} w\right]^{T}
$$

where

$$
C_{0}^{-1}=\frac{1}{\varepsilon_{11} \mu_{11}-d_{11}^{2}}\left[\begin{array}{cc}
-\mu_{11} & d_{11} \\
d_{11} & -\varepsilon_{11}
\end{array}\right]=\left[\begin{array}{ll}
c_{1} & c_{2} \\
c_{2} & c_{3}
\end{array}\right] .
$$

The relevant field variables are

$$
\begin{gathered}
\tau_{z k}=\tilde{c}_{44} w_{, k}-\alpha D_{k}-\beta B_{k}, \\
\phi=\alpha w+c_{1} \chi+c_{2} \eta, \\
\psi=\beta w+c_{2} \chi+c_{3} \eta, \\
D_{k}=\chi, k, \\
B_{k}=\eta_{, k}, \quad k=x, y, \\
\nabla^{2} w=0, \quad \nabla^{2} \chi=0, \quad \nabla^{2} \eta=0 .
\end{gathered}
$$

The two last equations (15) are equivalent to (9) since $c_{1} c_{3}-c_{2}^{2}=1 /\left(\varepsilon_{11} \mu_{11}-d_{11}^{2}\right) \neq 0$. In (14) the material parameters are defined as follows:

$$
\begin{gathered}
\tilde{c}_{44}=c_{44}+\alpha e_{15}+\beta q_{15}, \\
\alpha=\frac{\mu_{11} e_{15}-d_{11} q_{15}}{\varepsilon_{11} \mu_{11}-d_{11}^{2}}=-\left(c_{1} e_{15}+c_{2} q_{15}\right), \\
\beta=\frac{\varepsilon_{11} q_{15}-d_{11} e_{15}}{\varepsilon_{11} \mu_{11}-d_{11}^{2}}=-\left(c_{3} q_{15}+c_{2} e_{15}\right) .
\end{gathered}
$$

Note that $\tilde{c}_{44}$ is the piezo-electro-magnetically stiffened elastic constant.

Note also that the inverse of a matrix $C$ is defined by parameters $\alpha, \beta, \widetilde{c}_{44}$ and $c_{1}, c_{2}, c_{3}$ as follows:

$$
C^{-1}=\frac{1}{\tilde{c}_{44}}\left[\begin{array}{ccc}
1 & \alpha & \beta \\
\alpha & \alpha^{2}+\tilde{c}_{44} c_{1} & \alpha \beta+\tilde{c}_{44} c_{2} \\
\beta & \alpha \beta+\tilde{c}_{44} c_{2} & \beta^{2}+\tilde{c}_{44} c_{3}
\end{array}\right]
$$

and is the matrix generalized compliances of PEMO-elastic material. These material parameters will be appear in our solutions. 


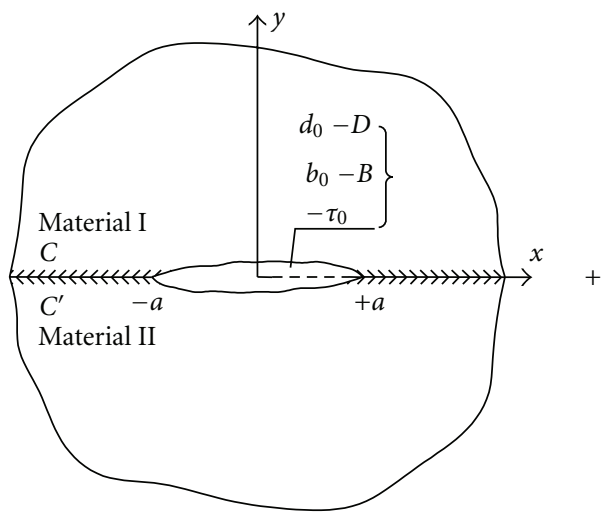

(a)

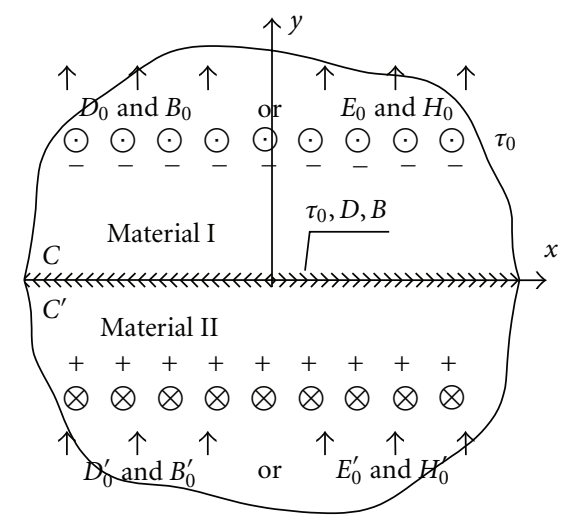

(b)

FiguRE 1: The interface crack under antiplane mechanical and in-plane electric and magnetic load. Inside the crack the unknown electromagnetic fields $d_{0}$ and $b_{0}$ appear. (a) Perturbation problem, (b) Elementary solution for bimaterial without the crack (19) and (20).

\section{Formulation of the Crack Problem}

Let the medium I occupy the upper half-space and medium II be in the lower half-space; the interface crack is assumed to be located in the region from $-a$ to $+a$ along the $x$-axis. The two-phase composite is subjected to electric, magnetic, and mechanical loads applied at infinity. These are $\left(\tau_{0}, D_{0}, B_{0}\right)$ or $\left(\tau_{0}, E_{0}, H_{0}\right)$. Under applied external loading, the crack, filled usually by vacuum or air, accumulated an electric and magnetic field, denoted by $d_{0}$ and $b_{0}$, would be built up. By the superposition principle, the interface crack problem is equivalent to the one under the applied loading on the upper surface (Figure 1):

$$
\begin{gathered}
\tau_{z y}(x, 0 \pm)=-\tau_{0}, \quad D_{y}(x, 0 \pm)=-D+d_{0}, \\
B_{y}(x, 0 \pm)=-B+b_{0} ; \quad|x|<a,
\end{gathered}
$$

where

$$
\begin{aligned}
& D_{y}=D \\
& = \begin{cases}D_{0}, & \text { case I } \\
\frac{e_{15}}{c_{44}} \tau_{0}+\left(\varepsilon_{11}+\frac{e_{15}^{2}}{c_{44}}\right) E_{0}+\left(d_{11}+\frac{e_{15} q_{15}}{c_{44}}\right) H_{0}, & \text { case II }\end{cases}
\end{aligned}
$$

$$
\begin{aligned}
& B_{y}=B \\
& = \begin{cases}B_{0}, & \text { case I } \\
\frac{q_{15}}{c_{44}} \tau_{0}+\left(d_{11}+\frac{e_{15} q_{15}}{c_{44}}\right) E_{0}+\left(\mu_{11}+\frac{q_{15}^{2}}{c_{44}}\right) H_{0}, & \text { case II. }\end{cases}
\end{aligned}
$$

Similarly is for lower crack surface, where the material parameters and electro-magnetic loadings are denoted by prime.

To guarantee the continuity of physical quantities at the perfectly bonded interface, applied electro-magnetic loadings $E$ and $H$ must obey the relations from which the loadings of upper material, namely, $E_{0}$ and $H_{0}$ may be determined by means of loading of lower material, namely, $E_{0}^{\prime}$ and $H_{0}^{\prime}$, using (19) and (20). Of course, $D_{0}=D_{0}^{\prime}$ and $B_{0}=B_{0}^{\prime}$ in Case $I$ of loading and $E_{0}=E_{0}^{\prime}$ and $H_{0}=H_{0}^{\prime}$ for homogeneous medium only.

At the interface $y=0 \pm$, we have the conditions

$$
\begin{array}{rlrl}
\left\lfloor\left|\tau_{z y}\right|\right\rfloor & =0, \quad\left\lfloor\left|D_{y}\right|\right\rfloor=0, \quad & \left\lfloor\left|B_{y}\right|\right\rfloor=0, \quad|x|<\infty, \\
{[|w|]=0,} & {[|\phi|]=0, \quad[|\psi|]=0, \quad|x| \geq a,}
\end{array}
$$

where the notation $[|f|]=f^{+}-f^{-}$and $f^{+}$denotes the value for $0^{+}$while $f^{-}$for $0^{-}$.

The electric displacement $d_{0}$ and magnetic induction $b_{0}$ inside the crack are obtained from semipermeable crack-face boundary conditions [5]. For two different magnetoelectric media: PEMO-elastic material I and notch space, we have the continuity condition of electric and magnetic potential in both materials at interfaces, similarly for interface between second PEMO-elastic material and crack interior. The semipermeable crack-face magnetoelectric boundary conditions are expressed as follows:

$$
d_{0}=-\varepsilon_{c} \frac{[|\phi|]}{2 \delta(x)}, \quad b_{0}=-\mu_{c} \frac{[|\psi|]}{2 \delta(x)},
$$

where $\delta(x)$ describes the shape of the notch, and $\varepsilon_{c}, \mu_{c}$ are the dielectric permittivity and magnetic permeability of crack interior. If we assume the elliptic notch profile such that

$$
\delta(x)=\frac{\delta_{0}}{a} \sqrt{a^{2}-x^{2}},
$$

where $\delta_{0}$ is the half-thickness of the notch at $x=0$, we obtain that

$$
\begin{gathered}
2 d_{0} \frac{\delta_{0}}{a \varepsilon_{c}} \sqrt{a^{2}-x^{2}}=-[|\phi|], \\
2 d_{0} \frac{\delta_{0}}{a \mu_{c}} \sqrt{a^{2}-x^{2}}=-[|\psi|] ; \quad|x| \leq a .
\end{gathered}
$$


Equations (24) form two coupling linear equations with respect to $d_{0}$ and $b_{0}$ since $[|\phi|]$ and $[|\psi|]$ depend linearly on these quantities as shown boundary conditions (18) and (21).

\section{The Solution for Two-Phase Medium with the Discontinuity at Interface}

Define the Fourier transform pair by the equations

$\hat{f}(s)=\int_{0}^{\infty} f(x) \cos (s x) d x, \quad f(x)=\frac{2}{\pi} \int_{0}^{\infty} \hat{f}(s) \cos (s x) d s$.

Then (15) are converted to ordinary differential equations and their solutions

$$
\begin{array}{lc}
\hat{w}(s, y)=A(s) e^{-s y}, & y \geq 0, \\
\hat{w}(s, y)=B(s) e^{s y}, & y \leq 0, \\
\hat{\chi}(s, y)=C(s) e^{-s y}, & y \geq 0, \\
\hat{\chi}(s, y)=-C(s) e^{s y}, & y \leq 0, \\
\hat{\psi}(s, y)=D(s) e^{-s y}, & y \geq 0, \\
\hat{\psi}(s, y)=-D(s) e^{s y}, & y \leq 0 .
\end{array}
$$

These solutions satisfy the regularity conditions at infinity and the conditions of vanishing jumps of electric displacement and magnetic induction at interface and crack surfaces.

From $(21)_{1}$ we obtain that

$$
B(s)=-\frac{\widetilde{c}_{44}}{\widetilde{c}_{44}^{\prime}} A(s)+\frac{\alpha-\alpha^{\prime}}{\widetilde{c}_{44}^{\prime}} C(s)+\frac{\beta-\beta^{\prime}}{\widetilde{c}_{44}^{\prime}} D(s)
$$

The material parameters of the lower material are denoted by prime.

The mixed boundary conditions on the crack plane and outside lead to

$$
\begin{aligned}
& \int_{0}^{\infty} s\left\{\begin{array}{l}
A(s) \\
C(s) \\
D(s)
\end{array}\right\} \cos (s x) d s=\left\{\begin{array}{c}
\frac{\tau_{0}+\alpha\left(D-d_{0}\right)+\beta\left(B-b_{0}\right)}{\widetilde{c}_{44}} \\
D-d_{0} \\
B-b_{0}
\end{array}\right\}, \\
& \int_{0}^{\infty} s\left\{\begin{array}{l}
A(s) \\
C(s) \\
D(s)
\end{array}\right\} \cos (s x) d s=\left\{\begin{array}{l}
0 \\
0 \\
0
\end{array}\right\}, \quad|x| \geq a .
\end{aligned}
$$

Using the integrals

$$
\begin{gathered}
\int_{0}^{\infty} J_{1}(a s) \cos (s x)=\frac{1}{a} \begin{cases}1 ; & |x|<a \\
a-\frac{|x|}{\sqrt{x^{2}-a^{2}}}, & |x| \geq a\end{cases} \\
\int_{0}^{\infty} \frac{J_{1}(a s)}{s} \cos (s x)= \begin{cases}0 ; & |x| \geq a \\
\frac{\sqrt{x^{2}-a^{2}}}{a}, & |x| \leq a\end{cases}
\end{gathered}
$$

we see that the solutions of (28) are

$$
\left\{\begin{array}{l}
A(s) \\
C(s) \\
D(s)
\end{array}\right\}=\frac{a}{s} J_{1}(a s)\left\{\begin{array}{c}
\frac{\tau_{0}+\alpha\left(D-d_{0}\right)+\beta\left(B-b_{0}\right)}{\widetilde{c}_{44}} \\
D-d_{0} \\
B-b_{0}
\end{array}\right\} .
$$

We calculate that

$$
\begin{aligned}
{[[|w|],[|\phi|],[|\psi|]]^{T}=} & \frac{2}{\pi} \sqrt{a^{2}-x^{2}}\left[C^{-1}+C^{\prime-1}\right] \\
& \times\left[\tau_{0}, D-d_{0}, B-b_{0}\right]^{T},
\end{aligned}
$$

where $C^{-1}$ is defined by the matrix (17) and $C^{\prime-1}$ by the same matrix with material parameters of second material.

From the condition (24), we obtain that

$$
\left[d_{0}, b_{0}\right]^{T}=-\frac{1}{2}\left[\begin{array}{cc}
\varepsilon_{0} & 0 \\
0 & \mu_{0}
\end{array}\right]\left[C_{1}^{-1}+C_{1}^{\prime-1}\right]\left[\tau_{0}, D-d_{0}, B-b_{0}\right]^{T},
$$

where

$$
\varepsilon_{0}=\frac{2}{\pi} \frac{a}{\delta_{0}} \varepsilon_{c}, \quad \mu_{0}=\frac{2}{\pi} \frac{a}{\delta_{0}} \mu_{c},
$$

$$
C_{1}^{-1}=\frac{1}{\tilde{c}_{44}}\left[\begin{array}{lll}
\alpha & \alpha^{2}+\tilde{c}_{44} c_{1} & \alpha \beta+\tilde{c}_{44} c_{2} \\
\beta & \alpha \beta+\tilde{c}_{44} c_{2} & \beta^{2}+\tilde{c}_{44} c_{3}
\end{array}\right]
$$

and similarly for $C_{1}^{\prime-1}$ (second material).

\section{Field Intensity Factors}

The singular behaviour of $\tau_{z y}, D_{y}$, and $B_{y}$ at $y=0,|x| \rightarrow a^{+}$ are:

$$
\left[\tau_{z y}, D_{y}, B_{y}\right]^{T}=\frac{2}{\pi} \frac{|x|}{\sqrt{x^{2}-a^{2}}}\left[\tau_{0}, D-d_{0}, B-b_{0}\right]^{T} .
$$

Defining the stress, electric displacement and magnetic induction intensity factors as follows:

$$
\left[K_{\tau}, K_{D}, K_{B}\right]^{T}=\lim _{|x| \rightarrow a^{+}} \sqrt{2(|x|-a)}\left[\tau_{z y}, D_{y}, B_{y}\right]^{T},
$$

we obtain that

$$
\left[K_{\tau}, K_{D}, K_{B}\right]^{T}=\frac{2}{\pi} \sqrt{a}\left[\tau_{0}, D-d_{0}, B-b_{0}\right]^{T} .
$$


Furthermore, we obtain the displacement, electric and magnetic potentials intensity factors

$$
\left[K_{w}, K_{\phi}, K_{\psi}\right]^{T}=\lim _{|x| \rightarrow a^{-}} \frac{1}{2 \sqrt{2(a-|x|)}}[[|w|],[|\phi|],[|\psi|]]^{T} .
$$

In view of results (31) and (37), we have

$$
\left[K_{w}, K_{\phi}, K_{\psi}\right]^{T}=\frac{1}{2}\left[C^{-1}+C^{\prime-1}\right]\left[K_{\tau}, K_{D}, K_{B}\right]^{T} .
$$

The energy release rate is defined as

$$
G=\frac{1}{2}\left(K_{\tau} K_{w}+K_{D} K_{\phi}+K_{B} K_{\psi}\right)
$$

and is the following:

$$
G=\frac{1}{4}\left[K_{\tau}, K_{D}, K_{B}\right]\left[C^{-1}+C^{\prime-1}\right]\left[K_{\tau}, K_{D}, K_{B}\right]^{T}
$$

or in explicit form

G

$$
\begin{aligned}
=\frac{1}{\pi^{2}} a\{( & \left.\frac{1}{\widetilde{c}_{44}}+\frac{1}{\widetilde{c}_{44}^{\prime}}\right) \tau_{0}^{2} \\
& +\left(\frac{\alpha^{2}}{\widetilde{c}_{44}}+\frac{\alpha^{\prime 2}}{\widetilde{c}_{44}^{\prime}}+c_{1}+c_{1}^{\prime}\right)\left(D-d_{0}\right)^{2} \\
& +\left(\frac{\beta^{2}}{\widetilde{\widetilde{c}_{44}}}+\frac{\beta^{\prime 2}}{\widetilde{c}_{44}^{\prime}}+c_{3}+c_{3}^{\prime}\right)\left(B-b_{0}\right)^{2} \\
& +2\left(\frac{\alpha}{\widetilde{c}_{44}}+\frac{\alpha^{\prime}}{\widetilde{c}_{44}^{\prime}}\right) \tau_{0}\left(D-d_{0}\right) \\
& +2\left(\frac{\beta}{\widetilde{c}_{44}}+\frac{\beta^{\prime}}{\widetilde{c}_{44}^{\prime}}\right) \tau_{0}\left(B-b_{0}\right) \\
& \left.+2\left(\frac{\alpha \beta}{\widetilde{c}_{44}}+\frac{\alpha^{\prime} \beta^{\prime}}{\widetilde{c}_{44}^{\prime}}+c_{2}+c_{2}^{\prime}\right)\left(D-d_{0}\right)\left(B-b_{0}\right)\right\} .
\end{aligned}
$$

For fully impermeable case, we have $d_{0}=0$ and $b_{0}=0$, and the solutions are obtained from (37) and (39). For fully permeable case, we have $\varepsilon_{0} \rightarrow \infty$ and $\mu_{0} \rightarrow \infty$ and

$D-d_{0}=\frac{1}{2}\left(\frac{e_{15}}{c_{44}}+\frac{e_{15}^{\prime}}{c_{44}^{\prime}}\right) \tau_{0}, \quad B-b_{0}=\frac{1}{2}\left(\frac{q_{15}}{c_{44}}+\frac{q_{15}^{\prime}}{c_{44}^{\prime}}\right) \tau_{0}$.

The energy release rate is

$$
G=\frac{1}{\pi^{2}}\left(\frac{1}{c_{44}}+\frac{1}{c_{44}^{\prime}}\right) \tau_{0}^{2} a .
$$

Note that energy release rate (44) for fully permeable crack problem is defined by the harmonic mean of the shear moduli of both materials, that is,

$$
G=\frac{2}{\pi^{2}} \frac{\tau_{0}^{2} a}{c_{44}^{*}} ; \quad \frac{1}{c_{44}^{*}}=\frac{1}{2}\left(\frac{1}{c_{44}}+\frac{1}{c_{44}^{\prime}}\right)
$$

The remaining field intensity factors are obtained, in this case, as follows:

$$
\begin{gathered}
K_{\tau}=\frac{2}{\pi} \tau_{0} \sqrt{a}, \quad K_{D}=\frac{1}{\pi}\left(\frac{e_{15}}{c_{44}}+\frac{e_{15}^{\prime}}{c_{44}^{\prime}}\right) \tau_{0} \sqrt{a}, \\
K_{B}=\frac{1}{\pi}\left(\frac{q_{15}}{c_{44}}+\frac{q_{15}^{\prime}}{c_{44}^{\prime}}\right) \tau_{0} \sqrt{a} .
\end{gathered}
$$

In particular, for fully permeable crack between two PEMO-elastic materials polarized in opposite directions, we have $K_{D}=0$ and $K_{B}=0$, since $e_{15}=-e_{15}^{\prime}$ and $q_{15}=-q_{15}^{\prime}$ in this case.

For electrically impermeable and magnetically permeable crack, the solutions are independent of the applied magnetic field $\left(d_{0}=0\right.$ and $B-b_{0}$ is independent on $B$ for $\varepsilon_{0} \rightarrow 0$ and $\mu_{0} \rightarrow \infty$ as shown in (32)).

Alternatively, the solutions for the electrically permeable and magnetically impermeable crack are independent on the applied electric displacement.

In practical applications the following cases appear:

(i) Let $\varepsilon_{0}$ tends to infinity and $\mu_{0}$ is finite.

Then

$$
\begin{aligned}
& K_{D}^{\text {perm } \cdot \mu_{c}}=K_{D}^{\text {perm } \cdot \text { imp }}\left[1-f_{1}(\bar{\mu})\right]+K_{D}^{\text {perm } \cdot \text { perm }} f_{1}(\bar{\mu}), \\
& K_{B}^{\text {perm } \cdot \mu_{c}}=K_{B}^{\text {imp } \cdot \text { imp }}\left[1-f_{1}(\bar{\mu})\right]+K_{B}^{\text {perm } \cdot \text { perm }} f_{1}(\bar{\mu}),
\end{aligned}
$$

where

$$
f_{1}(\bar{\mu})=\frac{1}{1+\bar{\mu}}, \quad \bar{\mu}=\frac{\pi}{2} \frac{\delta_{0}}{a} \frac{1}{\mu_{c}} \frac{1}{2}\left(\mu_{11}+\frac{q_{15}^{2}}{c_{44}}+\mu_{11}^{\prime}+\frac{q_{15}^{\prime 2}}{c_{44}^{\prime}}\right) .
$$

(ii) Let $\mu_{0}$ tends to infinity and $\varepsilon_{0}$ is finite.

Then

$$
\begin{aligned}
K_{D}^{\mathcal{\varepsilon}_{c}} \cdot \text { perm } & =K_{D}^{\text {imp } \cdot \text { imp }}\left[1-f_{2}(\bar{\varepsilon})\right]+K_{D}^{\text {perm } \cdot \text { perm }} f_{2}(\bar{\varepsilon}), \\
K_{B}^{\varepsilon_{c}} \cdot \text { perm } & =K_{B}^{\text {imp } \cdot \operatorname{perm}}\left[1-f_{2}(\bar{\varepsilon})\right]+K_{B}^{\text {perm } \cdot \text { perm }} f_{2}(\bar{\varepsilon}),
\end{aligned}
$$

where

$$
f_{2}(\bar{\varepsilon})=\frac{1}{1+\bar{\varepsilon}}, \quad \bar{\varepsilon}=\frac{\pi}{2} \frac{\delta_{0}}{a} \frac{1}{\varepsilon_{c}} \frac{1}{2}\left(\varepsilon_{11}+\frac{e_{15}^{2}}{c_{44}}+\varepsilon_{11}^{\prime}+\frac{e_{15}^{\prime 2}}{c_{44}^{\prime}}\right) .
$$

The functions of permittivity $\left(\varepsilon_{c}\right)$ and permeability $\left(\mu_{c}\right)$ approaches zero as $\varepsilon_{c}$ and $\mu_{c}$ tend to zero and are unity as $\varepsilon_{c}$ and $\mu_{c}$ tend to infinity. The solution perfectly matches exact solution in both limiting cases, namely, permeable and/or impermeable electric and/or magnetic boundary conditions.

In above equations the notation $K^{\text {perm.imp }}$ denotes the intensity factor for electrically permeable and magnetically impermeable crack boundary conditions.

The electric displacement $d_{0}$ and magnetic induction $b_{0}$ in the crack region depend on the matrix

$$
\bar{C}_{1}^{-1}=\frac{1}{2}\left(C_{1}^{-1}+C_{1}^{-1}\right)
$$


as well as $K_{D}, K_{B}, K_{\phi}, K_{\psi}$, and $K_{w}$ depend on the matrix

$$
\bar{C}^{-1}=\frac{1}{2}\left(C^{-1}+C^{\prime-1}\right)
$$

where again " -1 ” denotes the inverse matrix.

Thus,

$$
\bar{C}=\left[\begin{array}{ccc}
\bar{c}_{44} & \bar{e}_{15} & \bar{q}_{15} \\
\bar{e}_{15} & -\bar{\varepsilon}_{11} & -\bar{d}_{11} \\
\bar{q}_{15} & -\bar{d}_{11} & -\bar{\mu}_{11}
\end{array}\right]=\left[\frac{1}{2}\left(C^{-1}+C^{\prime-1}\right)\right]^{-1}
$$

is the matrix of material property of equivalent homogeneous material after homogenization in our problem. The generalized effective electroelastic compliances of bi-material system are obtained as arithmetically mean of compliances of single materials constituents. If the lower medium and the upper medium have the same properties but are poled in opposite directions, then $\alpha=-\alpha^{\prime}$ and $\beta=-\beta^{\prime}$ (see (16)). In consequence from (17), we have

$$
\bar{C}^{-1}=\frac{1}{\widetilde{c}_{44}}\left[\begin{array}{ccc}
1 & 0 & 0 \\
0 & \alpha^{2}+c_{1} \tilde{c}_{44} & \alpha \beta+c_{2} \tilde{c}_{44} \\
0 & \alpha \beta+c_{2} \tilde{c}_{44} & \beta^{2}+c_{3} \tilde{c}_{44}
\end{array}\right]
$$

Then

$$
\begin{gathered}
\bar{C}=\frac{\tilde{c}_{44}}{\Delta}\left[\begin{array}{ccc}
\Delta & 0 & 0 \\
0 & \beta^{2}+c_{3} \tilde{c}_{44} & -\left(\alpha \beta+c_{2} \tilde{c}_{44}\right) \\
0 & -\left(\alpha \beta+c_{2} \tilde{c}_{44}\right) & \alpha^{2}+c_{1} \tilde{c}_{44}
\end{array}\right], \\
\Delta=\left(\alpha^{2}+c_{1} \tilde{c}_{44}\right)\left(\beta^{2}+c_{3} \tilde{c}_{44}\right)-\left(\alpha \beta+c_{2} \tilde{c}_{44}\right)^{2} .
\end{gathered}
$$

Magneto-electro-elastic materials usually comprise alternating piezoelectric material and piezomagnetic material. If upper material is piezomagnetic and lower material is piezoelectric (or otherwise), we have

$$
C=\left[\begin{array}{ccc}
c_{44} & 0 & q_{15} \\
0 & -\infty & 0 \\
q_{15} & 0 & -\mu_{11}
\end{array}\right], \quad C^{\prime}=\left[\begin{array}{ccc}
c_{44}^{\prime} & e_{15}^{\prime} & 0 \\
e_{15}^{\prime} & -\varepsilon_{11}^{\prime} & 0 \\
0 & 0 & -\infty
\end{array}\right]
$$

The bi-material matrix $\bar{C}^{-1}$ defined by (51) has the form

$$
\bar{C}^{-1}=\frac{1}{2} \frac{\left[\begin{array}{ccc}
\mu_{11} & 0 & q_{15} \\
0 & 0 & 0 \\
q_{15} & 0 & -c_{44}
\end{array}\right]}{c_{44} \mu_{11}+q_{15}^{2}}+\frac{1}{2} \frac{\left[\begin{array}{ccc}
\varepsilon_{11}^{\prime} & e_{15}^{\prime} & 0 \\
e_{15}^{\prime} & -c_{44}^{\prime} & 0 \\
0 & 0 & 0
\end{array}\right]}{c_{44}^{\prime} \varepsilon_{11}^{\prime}+e^{\prime 2}}
$$

In the solutions also appears electric and magnetic field components

$$
\begin{array}{r}
{\left[E_{y}, H_{y}\right]^{T}=-C_{1}^{-1}\left[\tau_{0}, D, B\right]^{T} \quad \text { in case I, }} \\
E_{y}=E_{0}, \quad H_{y}=H_{0} \quad \text { in case II, }
\end{array}
$$

where the matrix $C_{1}^{-1}$ is defined by (34). Of course, for lower material, we have $C_{1}^{-1}$ matrix (the material parameters are denoted by prime). This states that, in general, electric and magnetic fields are also singular. The electric and magnetic field intensity factors $K_{E}$ and $K_{H}$ are related to $K_{\tau}, K_{D}$, and $K_{B}$, as shown $(57)_{1}$. In particular, for a fully permeable crack between two materials polarized in opposite directions, we have $K_{D}=0=K_{B}$ and

$$
K_{E}=\frac{\alpha}{\widetilde{c}_{44}} K_{\tau}, \quad K_{H}=\frac{\beta}{\widetilde{c}_{44}} K_{\tau}
$$

The particular solutions

$$
\begin{array}{cc}
w(y)=\gamma y, & w^{\prime}(y)=\gamma^{\prime} y, \\
\phi(y)=-E_{y} y, & \phi^{\prime}(y)=-E_{y}^{\prime} y, \\
\psi(y)=-H_{y} y, & \psi^{\prime}(y)=-H_{y}^{\prime} y
\end{array}
$$

with

$$
\gamma=\frac{1}{\widetilde{c}_{44}}\left(\tau_{0}+\alpha D+\beta B\right), \quad \gamma^{\prime}=\frac{1}{\widetilde{c}_{44}^{\prime}}\left(\tau_{0}+\alpha^{\prime} D+\beta^{\prime} B\right)
$$

complete the full fields in both materials.

\section{Numerical Results}

The basic data for the material properties selected here are similar to those in Sih and Song $[6,7]$. These constants read as: $c_{44}=43,7 \times 10^{9} \mathrm{~N} / \mathrm{m}^{2}, e_{15}=8,12 \mathrm{C} / \mathrm{m}^{2}, \varepsilon_{11}=$ $7,86 \times 10^{-9} \mathrm{C} / \mathrm{Vm}, d_{11}=0,0, q_{15}=165,0 \mathrm{~N} / \mathrm{Am}$, and $\mu_{11}=180,5 \times 10^{-6} \mathrm{~N} / \mathrm{A}^{2}$ for first material and $c_{44}=44,6 \times$ $10^{9} \mathrm{~N} / \mathrm{m}^{2}, e_{15}=3,48 \mathrm{C} / \mathrm{m}^{2}, \varepsilon_{11}=3,42 \times 10^{-9} \mathrm{C} / \mathrm{Vm}, d_{11}=$ $0,0, q_{15}=385,0 \mathrm{~N} / \mathrm{Am}, \mu_{11}=414,5 \times 10^{-6} \mathrm{~N} / \mathrm{A}^{2}$ for second material.

Using these properties of both materials, the material property matrix $\bar{C}^{-1}$ is obtained as (the matrix of generalized “compliances"):

$$
\bar{C}^{-1}=\left[\begin{array}{ccc}
19,88 \times 10^{-12} \mathrm{~m}^{2} / \mathrm{N} & 20,38 \times 10^{-3} \mathrm{~m}^{2} / \mathrm{C} & 18,33 \times 10^{-6} \mathrm{Am} / \mathrm{N} \\
20,38 \times 10^{-3} \mathrm{~m}^{2} / \mathrm{C} & -1,89 \times 10^{8} \mathrm{Vm} / \mathrm{C} & 18,78 \times 10^{3} \mathrm{Am} / \mathrm{C} \\
18,33 \times 10^{-6} \mathrm{Am} / \mathrm{N} & 18,78 \times 10^{3} \mathrm{Am} / \mathrm{C} & -3,96 \times 10^{3} \mathrm{~A}^{2} / \mathrm{N}
\end{array}\right]
$$


The matrix of generalized stiffness is obtained as follows:

$$
\bar{C}=\left[\begin{array}{ccc}
45,08 \times 10^{9} \mathrm{~N} / \mathrm{m}^{2} & 4,89 \mathrm{C} / \mathrm{m}^{2} & 231,8 \mathrm{~N} / \mathrm{Am} \\
4,89 \mathrm{C} / \mathrm{m}^{2} & -4,77 \times 10^{-9} \mathrm{C} / \mathrm{Vm} & 1,36 \times 10^{-12} \mathrm{C} / \mathrm{Am} \\
231,8 \mathrm{~N} / \mathrm{Am} & 1,36 \times 10^{-12} \mathrm{C} / \mathrm{Am} & -251 \times 10^{-6} \mathrm{~N} / \mathrm{A}^{2}
\end{array}\right] .
$$

Therefore, the properties of composite, obtained by averaging the properties of single-phase materials using its volume fractions, as in the literature (see [8]) gives erroneous result, since give (if ratio is roughly $50: 50$ )

$$
\bar{C}_{\text {aver }}=\left[\begin{array}{ccc}
44,15 \times 10^{9} \mathrm{~N} / \mathrm{m}^{2} & 5,80 \mathrm{C} / \mathrm{m}^{2} & 275 \mathrm{~N} / \mathrm{Am} \\
5,80 \mathrm{C} / \mathrm{m}^{2} & -5,64 \times 10^{-9} \mathrm{C} / \mathrm{Vm} & 0 \\
275 \mathrm{~N} / \mathrm{Am} & 0 & -297,5 \times 10^{-6} \mathrm{~N} / \mathrm{A}^{2}
\end{array}\right] .
$$

The nonzero material constants for $\mathrm{BaTiO}_{3}$-piezoelectric and $\mathrm{CoFe}_{2} \mathrm{O}_{4}$-magnetostrictive medium are given in Table 1 [9].
The bi-material matrix $\bar{C}^{-1}$ defined by (51) is ("compliance" matrix)

$$
\bar{C}^{-1}=\left[\begin{array}{ccc}
20,00 \times 10^{-12} \mathrm{~m}^{2} / \mathrm{N} & 94,13 \times 10^{-4} \mathrm{~m}^{2} / \mathrm{C} & 10,17 \times 10^{-6} \mathrm{Am} / \mathrm{N} \\
94,13 \times 10^{-4} \mathrm{~m}^{2} / \mathrm{C} & -6,28 \times 10^{9} \mathrm{Vm} / \mathrm{C} & 0 \\
10,17 \times 10^{-6} \mathrm{Am} / \mathrm{N} & 0 & -1,01 \times 10^{5} \mathrm{~A}^{2} / \mathrm{N}
\end{array}\right] .
$$

The matrix $\bar{C}$ is obtained as follows: ("stiffness" matrix)

$$
\bar{C}=\left[\begin{array}{ccc}
49,96 \times 10^{9} \mathrm{~N} / \mathrm{m}^{2} & 74,82 \times 10^{-3} \mathrm{C} / \mathrm{m}^{2} & 5,04 \mathrm{~N} / \mathrm{Am} \\
74,82 \times 10^{-3} \mathrm{C} / \mathrm{m}^{2} & -1,59 \times 10^{-10} \mathrm{C} / \mathrm{Vm} & 7,55 \times 10^{-12} \mathrm{C} / \mathrm{Am} \\
5,04 \mathrm{~N} / \mathrm{Am} & 7,55 \times 10^{-12} \mathrm{C} / \mathrm{Am} & -9,91 \times 10^{-6} \mathrm{~N} / \mathrm{A}^{2}
\end{array}\right] .
$$

Using the mixture rule [6], $\kappa^{c}=\kappa V_{f}+\kappa^{\prime}\left(1-V_{f}\right)$, for $V_{f}=0,5$, where $\kappa$ with superscripts $c$ without prime or prime denotes the corresponding constants $c_{44}, e, \varepsilon, q, \mu, d$ of the composite, first and second material, respectively, and $V_{f}$ is the volume fraction of the first material (piezoelectric), we obtain that

$$
\bar{C}^{*}=\left[\begin{array}{ccc}
44,15 \times 10^{9} \mathrm{~N} / \mathrm{m}^{2} & 5,80 \mathrm{C} / \mathrm{m}^{2} & 275 \mathrm{~N} / \mathrm{Am} \\
5,80 \mathrm{C} / \mathrm{m}^{2} & -5,64 \times 10^{-9} \mathrm{C} / \mathrm{Vm} & 0 \\
275 \mathrm{~N} / \mathrm{Am} & 0 & -297,5 \times 10^{-6} \mathrm{~N} / \mathrm{A}^{2}
\end{array}\right]
$$

which completely differs from $\bar{C}$.

Note that in both examples the sums of corresponding material parameters are constant. In consequence the matrix, $\bar{C}_{\text {aver }}$ and $\bar{C}^{*}$ have the same elements. Of course, the matrices of generalized stiffness are dissimilar in both bi-material composites.
Due to the absence of magnetoelectric coupling coefficient in a single-phase piezoelectric and piezomagnetic material, the magnetoelectric constant $d_{11}$, existing only in the piezoelectric/piezomagnetic composite as a significant new feature, cannot be determined by the above mixture rule. Therefore, based on the analysis of micromechanics, 
TABLE 1: The material constants for $\mathrm{BaTiO}_{3}$ and $\mathrm{CoFe}_{2} \mathrm{O}_{4}$.

\begin{tabular}{lcc}
\hline properties & $\mathrm{BaTiO}_{3}$ piezoelectric & $\mathrm{CoFe}_{2} \mathrm{O}_{4}$ piezomagnetic \\
\hline$c_{44}\left(10^{9} \mathrm{~N} / \mathrm{m}^{2}\right)$ & 43,00 & 45,30 \\
$e_{15}\left(\mathrm{C} / \mathrm{m}^{2}\right)$ & 11,60 & 0,00 \\
$\varepsilon_{11}\left(10^{-9} \mathrm{C} / \mathrm{Vm}\right)$ & 11,20 & 0,08 \\
$q_{15}(\mathrm{~N} / \mathrm{Am})$ & 0,00 & 550,00 \\
$\mu_{11}\left(10^{-6} \mathrm{~N} / \mathrm{A}^{2}\right)$ & 5,00 & 590,00 \\
$d_{11}\left(10^{-9} \mathrm{C} / \mathrm{Am}\right)$ & 0,00 & 0,00 \\
\hline
\end{tabular}

this coefficient is obtained as $d_{11}=1,36 \times 10^{-12} \mathrm{C} / \mathrm{Am}$ for first combination of materials and 7,55 $\times 10^{-12} \mathrm{C} / \mathrm{Am}$ for barium titanate-cobalt iron oxide bi-material. This is magnetoelectric coupling effect in composite of piezoelectric and piezomagnetic phases.

\section{Conclusions}

The mode III interface crack in a bi-material magnetoelectro-elastic medium subjected to mechanical, electrical, and magnetic loads on the surfaces is studied in this paper, and the following points are noted.

(i) Closed form solution has been obtained for a crack between two dissimilar PEMO-elastic materials. Expressions for the crack-tip field intensity factors, the electromagnetic fields inside the crack, are given. The semipermeable crack-face magnetoelectric boundary conditions are investigated.

(ii) The energy release rate can be explicitly expressed in terms of the external loadings (by (42)). It is affected by electric-magnetic properties of the two constituents of the bi-material media.

(iii) Applications of electric and magnetic fields do not alter the stress intensity factor of mode III. The values of SIF are identical for any kind of crack-face electric and magnetic boundary condition assumptions. In other words, the crack-face electric and magnetic boundary conditions have no effects on SIF.

(iv) For electrically impermeable and magnetically permeable crack, the solutions for field intensity factors are independent of the applied magnetic field. Alternatively, these solutions for the electrically permeable and magnetically impermeable crack are independent on the applied electric displacement.

(v) For fully permeable crack between two PEMOelastic materials polarized in opposite directions, the electric displacement and magnetic induction intensity factors vanish. In this case electric and magnetic field intensity factors $K_{E}$ and $K_{B}$ are related to $K_{\tau}$ (by $(58)$ ).

(vi) The matrices of "generalized" compliances or stiffness cannot be determined by the mixture rule since it is a significant new feature in interface crack problem considered in this paper. (vii) From the reviewing of literature dealing with interface crack, problem may be concluded that the characterization of bonded dissimilar materials with interface crack is still an open problem.

\section{References}

[1] R. Li and G. A. Kardomateas, "The mode III interface crack in piezo-electro-magneto-elastic dissimilar bimaterials," Journal of Applied Mechanics, vol. 73, no. 2, pp. 220-227, 2006.

[2] B. L. Wang and Y. W. Mai, "Closed-form solution for an antiplane interface crack between two dissimilar magnetoelectroelastic layers," Journal of Applied Mechanics, vol. 73, no. 2, pp. 281-290, 2006.

[3] X. F. Li and B. L. Wang, "Anti-plane shear crack normal to and terminating at the interface of two bonded piezoelectric ceramics," International Journal of Solids and Structures, vol. 44, no. 11-12, pp. 3796-3810, 2007.

[4] B. Rogowski, "The mode III cracks emanating from an elliptical hole in the piezo-electro-magneto-elastic materials," Archive of Applied Mechanics, vol. 81, no. 11, pp. 1607-1620, 2011.

[5] B. Rogowski, "The limited electrically permeable crack model in linear piezoelasticity," International Journal of Pressure Vessels and Piping, vol. 84, no. 9, pp. 572-581, 2007.

[6] G. C. Sih and Z. F. Song, "Magnetic and electric poling effects associated with crack growth in $\mathrm{BaTiO}_{3}-\mathrm{CoFe}_{2} \mathrm{O}_{4}$ composite," Theoretical and Applied Fracture Mechanics, vol. 39, no. 3, pp. 209-227, 2003.

[7] Z. F. Song and G. C. Sih, "Crack initiation behavior in magnetoelectroelastic composite under in-plane deformation," Theoretical and Applied Fracture Mechanics, vol. 39, no. 3, pp. 189-207, 2003.

[8] B. L. Wang and Y. W. Mai, "Applicability of the crack-face electromagnetic boundary conditions for fracture of magnetoelectroelastic materials," International Journal of Solids and Structures, vol. 44, no. 2, pp. 387-398, 2007.

[9] J. H. Huang and W. S. Kuo, "The analysis of piezoelectric/piezomagnetic composite materials containing ellipsoidal inclusions," Journal of Applied Physics, vol. 81, no. 3, pp. 13781386, 1997. 

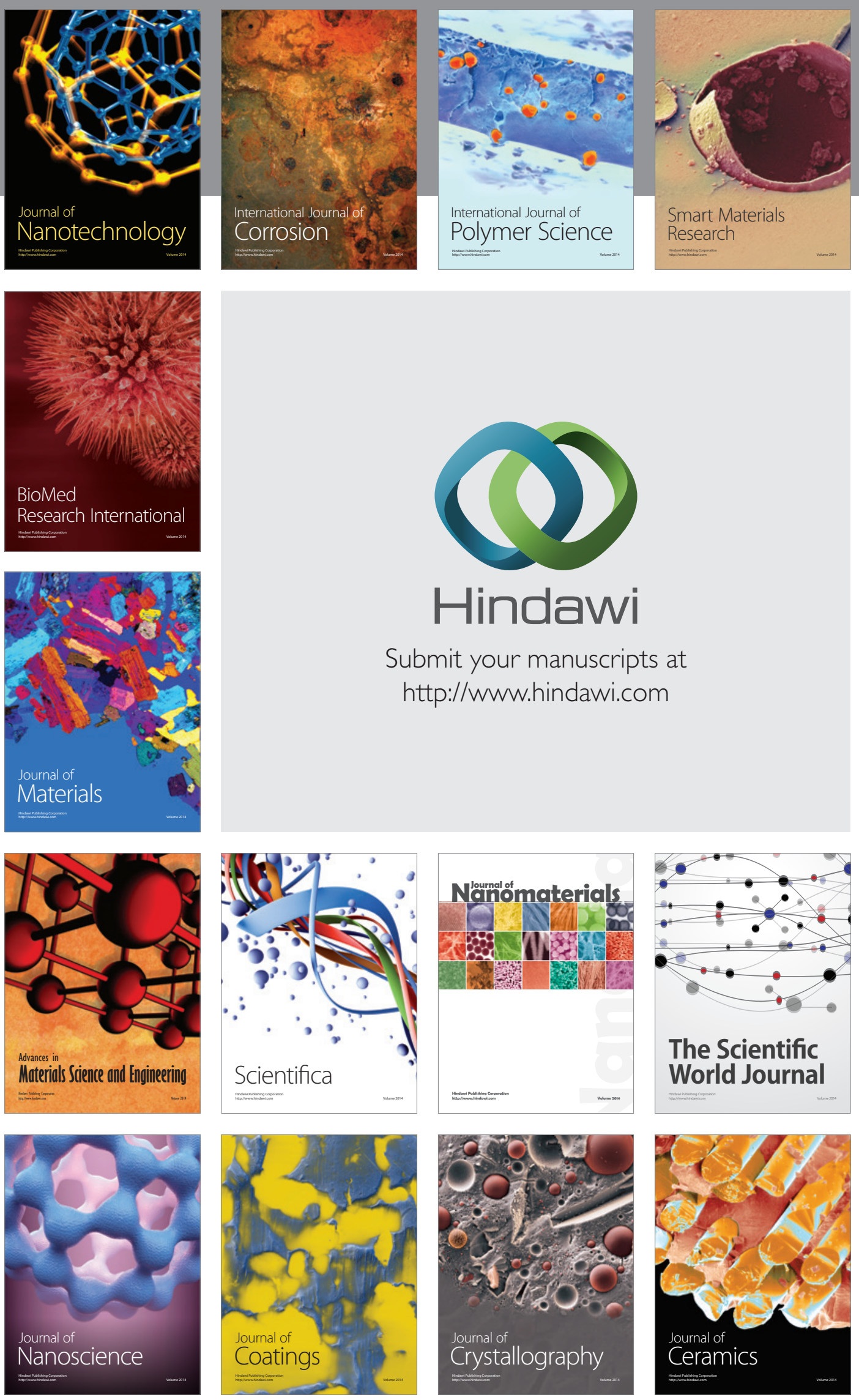

The Scientific World Journal

Submit your manuscripts at

http://www.hindawi.com

\section{World Journal}

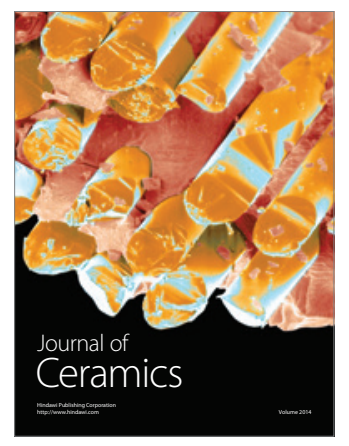

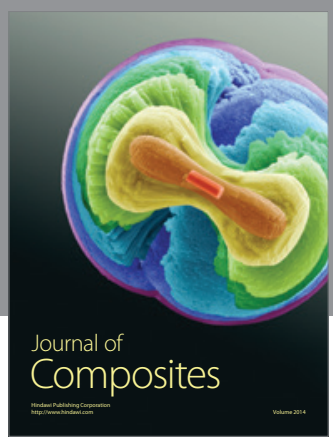
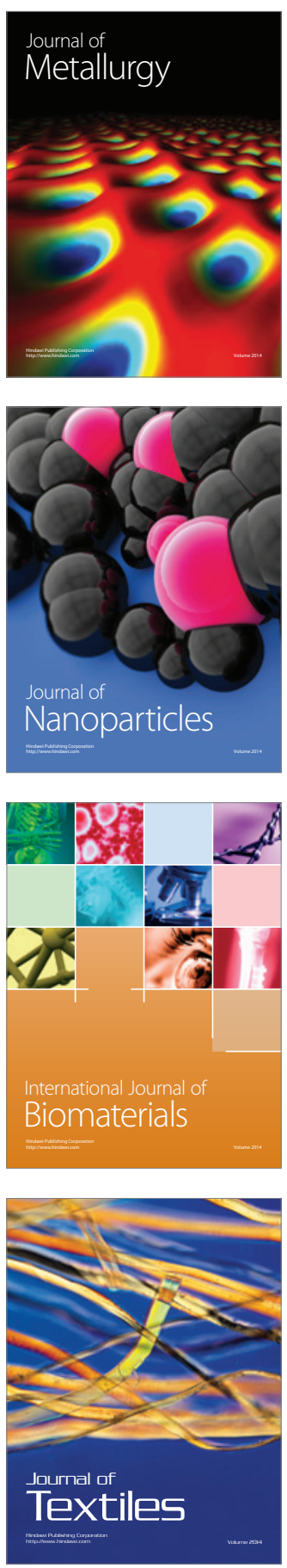